\title{
Peso del Corazón y Pulmones en Relación al Sexo, Grupo Étnico, Edad e Índice de Masa Corporal en Individuos Chilenos
}

\author{
Weight of the Heart and Lungs with Regard to Sex, \\ Ethnic Group, Age and Body Mass Index in Chilean Individuals
}

Olave E.*; Torrez, J. C.*; Riquelme, N.**; Ibacache, L.** \& Binvignat, O.***

OLAVE, E.; TORREZ, J. C.; RIQUELME, N.; IBACACHE, L. \& BINVIGNAT, O. Peso del corazón y pulmones en relación al sexo, grupo étnico, edad e índice de masa corporal en individuos chilenos. Int. J. Morphol., 32(3):866-870, 2014.

RESUMEN: Diversos factores pueden influir en el peso de las vísceras torácicas y/o abdominales. Con el propósito de conocer el peso promedio del corazón y pulmones de individuos chilenos, se obtuvo la información de estos órganos procedentes de 775 necropsias de cadáveres frescos, entre los años 2009-2013, considerando su procedencia étnica (Mapuches y no Mapuches), edad, sexo e índice de masa corporal. Se estudiaron los datos de órganos sin lesiones ni enfermedades aparentes. En relación a la edad, el peso promedio del corazón se incrementó en ambos grupos étnicos y ambos sexos. Este incremento fue similar en los individuos de sexo masculino de ambos grupos étnicos, con un valor de 13\%. En los individuos de sexo femenino hubo un aumento de 36\% en el grupo étnico Mapuche, mientras que en el grupo No-Mapuche fue de un $8 \%$. Con respecto a los pulmones, no hubo incremento de su peso promedio en individuos masculinos del grupo Mapuche, pero si lo hubo en el sexo femenino, donde hubo un incremento sobre el $40 \%$ Entre el grupo de 20-29 años y el de 30-39 años, ambos pulmones aumentaron sus pesos promedios en 35\%. En el grupo No-Mapuche, en ambos sexos, el peso promedio de estos órganos aumentó menos de un 10\%. Estos resultados son comparados con otros grupos poblacionales del mundo y son un aporte al conocimiento morfológico de las vísceras torácicas.

PALABRAS CLAVE: Anatomía; Tórax; Peso visceral; Corazón; Pulmones.

\section{INTRODUCCIÓN}

Muchos factores pueden influir en el peso de los diversos órganos del cuerpo, entre los cuales tenemos el sexo, la edad, el índice de masa corporal y el grupo étnico al que pertenecen. Un peso excesivo de algunos de estos órganos puede derivar en diversas dolencias que afectan a las personas y a su calidad de vida.

En algunos órganos, existen controversias con respecto a la influencia de la edad, como es el caso del peso del corazón. Autores como Smith (1928) y Hodkinson et al. (1979) concluyeron que el peso del corazón no se incrementa con la edad. Sin embargo, Kitzman et al. (1988), de Suarez \& Avilán (2004) y Sheikhazadi et al. (2010) han señalado un aumento en el peso promedio del corazón, con la diferencia que los primeros indicaron que sólo ocurrió este cambio en mujeres entre 20 y 69 años.

\footnotetext{
" Facultad de Medicina, Universidad de La Frontera, Temuco, Chile.

** Universidad Mayor, Temuco, Chile.

**** Universidad Autónoma de Chile, Sede Talca, Chile.
} 
altura de cada individuo, el peso del corazón y pulmones. Se agrupó por etnia, edad, sexo e índice de masa corporal, calculando estadísticamente el promedio, la desviación estándar y asociando el peso promedio de cada una de las vísceras con estos parámetros.

\section{RESULTADOS}

En el grupo no-Mapuche, el corazón tuvo un peso promedio de 391,2 g y 300 g, en hombres y mujeres, respectivamente; en el hombre, el peso del pulmón derecho e izquierdo fue $575 \mathrm{~g}$ and 503,2 $\mathrm{g}$, respectivamente $\mathrm{y}$ en la mujer fue de 449,4 g y 386,3 g, respectivamente.
En el grupo de individuos Mapuches, el corazón tuvo un peso promedio de $386 \mathrm{~g}$ y $280,2 \mathrm{~g}$, en hombre y mujeres, respectivamente; en el hombre, el peso del pulmón derecho e izquierdo fue de 575,8 g and 494,9 g, respectivamente y en la mujer de 409,4 g y 350,4 g, respectivamente.

En relación a grupos etarios e IMC los resultados obtenidos son presentados en Tablas. En la Tabla I se muestran los pesos promedios del corazón y pulmones de individuos Chilenos Mapuches, de ambos sexos, de acuerdo a la edad y en la Tabla II de individuos del grupo no-Mapuche. Respecto a la relación del peso de estos órganos con el IMC, la Tabla III presenta muestra los resultados promedios en individuos Mapuches de ambos sexos y la Tabla IV de individuos no-Mapuches.

Tabla I. Peso (g) del corazón y pulmones en hombres y mujeres en relación a la edad en individuos Mapuches.

\begin{tabular}{lcccccc}
\hline \multirow{2}{*}{ Edad (años) } & Hombres & \multicolumn{5}{c}{ Mujeres } \\
\cline { 2 - 7 } & Corazón & Pulmón der. & Pulmón izq. & Corazón & Pulmón der. & Pulmón izq. \\
\hline $20-29$ & $357,8 \pm 57,5$ & $577 \pm 121,5$ & $497 \pm 131,1$ & $236,5 \pm 51,2$ & $344,2 \pm 117$ & $283,5 \pm 103,9$ \\
$30-39$ & $379,3 \pm 65,9$ & $591,7 \pm 163,4$ & $510,6 \pm 139,5$ & $272,8 \pm 43,5$ & $464,4 \pm 96,6$ & $389,7 \pm 93,3$ \\
$40-49$ & $391,9 \pm 75,7$ & $592,4 \pm 196,4$ & $493,3 \pm 170,8$ & $315,3 \pm 76,3$ & $381,7 \pm 97,1$ & $324 \pm 89,6$ \\
$50-59$ & $404,4 \pm 62,6$ & $557,4 \pm 199,8$ & $487,7 \pm 173,6$ & $321,3 \pm 45,7$ & $486,8 \pm 154,9$ & $457 \pm 174,3$ \\
\hline
\end{tabular}

Tabla II. Peso (g) del corazón y pulmones en hombres y mujeres en relación a la edad en individuos No-Mapuches.

\begin{tabular}{lcccccc}
\hline \multirow{2}{*}{ Edad (años) } & Hombres & \multicolumn{5}{c}{ Mujeres } \\
\cline { 2 - 7 } & Corazón & Pulmón der. & Pulmón izq. & Corazón & Pulmón der. & Pulmón izq. \\
\hline $20-29$ & $361,4 \pm 62,8$ & $534,4 \pm 185,7$ & $472,7 \pm 153,7$ & $288,3 \pm 68,9$ & $464,8 \pm 142,6$ & $407,2 \pm 180,8$ \\
$30-39$ & $386,2 \pm 81,8$ & $588,2 \pm 288,3$ & $505,1 \pm 164,2$ & $283,4 \pm 47,6$ & $525,1 \pm 160,8$ & $462,4 \pm 126,8$ \\
$40-49$ & $396,2 \pm 72,6$ & $600,2 \pm 210,1$ & $525 \pm 212,8$ & $305,3 \pm 60,2$ & $402,9 \pm 173$ & $326,5 \pm 152,9$ \\
$50-59$ & $411,3 \pm 75$ & $575,1 \pm 179,4$ & $510,6 \pm 171,4$ & $311,6 \pm 59,4$ & $429,1 \pm 150,5$ & $370,7 \pm 134$ \\
\hline
\end{tabular}

Tabla III. Peso promedio (g) del corazón y pulmones en individuos Mapuches en relación al índice de masa corporal (IMC).

\begin{tabular}{llllllr}
\hline \multirow{2}{*}{ IMC } & \multicolumn{1}{c}{ Hombres } & \multicolumn{5}{c}{ Mujeres } \\
\cline { 2 - 7 } & \multicolumn{1}{c}{ Corazón } & \multicolumn{1}{c}{ Pulmón der. } & \multicolumn{1}{c}{ Pulmón izq. } & \multicolumn{1}{c}{ Corazón } & \multicolumn{1}{c}{ Pulmón der. } & Pulmón izq. \\
\hline $18,1-25$ & $354,2 \pm 64,6$ & $565,3 \pm 153,6$ & $483 \pm 135,9$ & $262,9 \pm 55,8$ & $374,7 \pm 97,2$ & $325,2 \pm 107,8$ \\
$25,1-30$ & $401,2 \pm 54,3$ & $588,9 \pm 172,8$ & $503,3 \pm 148$ & $265 \pm 48,5$ & $396,8 \pm 157,8$ & $322,5 \pm 127,4$ \\
$30,1-35$ & $414,4 \pm 65,5$ & $568,7 \pm 208,4$ & $482,4 \pm 195,8$ & --- & --- & --- \\
\hline
\end{tabular}

Tabla IV. Peso promedio (g) del corazón y pulmones en individuos no-Mapuches en relación al índice de masa corporal (IMC).

\begin{tabular}{lcccccc}
\hline \multirow{2}{*}{ IMC } & Hombres & \multicolumn{5}{c}{ Mujeres } \\
\cline { 2 - 7 } & Corazón & Pulmón der. & Pulmón izq. & Corazón & Pulmón der. & Pulmón izq. \\
\hline $18,1-25$ & $360,6 \pm 54$ & $568,8 \pm 182,1$ & $503,4 \pm 163,3$ & $282,8 \pm 51,7$ & $460 \pm 151,1$ & $403,9 \pm 166,9$ \\
$25,1-30$ & $399,5 \pm 62,5$ & $587,2 \pm 241,4$ & $496 \pm 174,9$ & $305,8 \pm 67$ & $459,8 \pm 164,8$ & $395,4 \pm 145,5$ \\
$30,1-35$ & $430,3 \pm 71$ & $574,1 \pm 221,4$ & $514,5 \pm 170,2$ & $322,5 \pm 57,7$ & $394,4 \pm 147,1$ & $330,5 \pm 120,1$ \\
$35,1-40$ & $458,1 \pm 58,6$ & $584 \pm 197$ & $515,6 \pm 151,1$ & --- & --- & -- \\
\hline
\end{tabular}




\section{DISCUSIÓN}

El peso de las vísceras torácicas y abdominales puede variar por diversos factores, no considerando enfermedades, entre los cuales podemos mencionar el sexo, la edad, el grupo étnico, etc. (Ruiz-Montero et al., 2013)

En la literatura clásica se describe que el peso del corazón en adultos es de 270 a 275 g, señalando que en la mujer suele ser en promedio de $250 \mathrm{~g}$ (Testut \& Latarjet, 1969). Por su parte, Gardner et al. (1977) han señalado que el peso medio de este órgano en varones es de $328 \mathrm{~g}$ con variaciones entre 256 -390 g y en la mujer de $244 \mathrm{~g}$, con variaciones entre 198-279 g. Otros autores han descrito que el peso medio del corazón en los hombres es de $300 \mathrm{~g}$ con variaciones entre 280-340 g y en la mujer un promedio de $250 \mathrm{~g}$ con variaciones entre 230 y $280 \mathrm{~g}$ (Williams et al., 1995). Por su parte Molina \& DiMaio (2012a) obtuvieron en hombres un peso promedio de $331 \mathrm{~g}$, con variaciones entre 188 y 575 g. Los pesos promedios obtenidos en nuestra serie se asemejan a estos últimos autores y a los informados por Kim et al. (2009), quienes indicaron promedios de 346,8 g para el hombre y 298,8 g para la mujer, señalando que hubo una correlación estadística significativa entre la edad y el peso de este órgano, y, que la altura y el peso corporal también influyen a los cambios de peso que presenta el corazón. Sin embargo, Molina \& DiMaio (2012a) señalaron que no encontraron relación entre el peso del corazón y el peso corporal, así como tampoco con la altura del cuerpo e IMC.

Autores como Smith y Hodkinson et al. concluyeron que el peso del corazón no se incrementa con la edad, conclusión también señalada por Gardner et al., explicando que la edad parece no tener influencia en el peso del miocardio, pero la cantidad de grasa epicárdica aumenta con el tiempo. Sin embargo, Kitzman et al., de Suarez \& Avilán y Sheikhazadi et al. han señalado un aumento en el peso promedio del corazón.

Nuestros resultados concuerdan con estos últimos autores, ya que se registró un aumento del peso promedio del corazón en individuos Mapuches y no-Mapuches, donde se apreció en ambos grupos que este órgano incrementó su peso promedio en un $13 \%$ en los individuos de sexo masculino, considerando una edad desde 20 a 59 años. Aún más, en los individuos de sexo femenino, el peso promedio se incrementó en las personas del grupo étnico Mapuche en un $35,8 \%$, considerando el peso inicial del grupo de 20 a 29 años con respecto al grupo de 50 a 59 años, en cambio el incremento entre estos grupos etarios fue de sólo un 8,1\% en las mujeres no-Mapuches.
Por su parte, Narongchai \& Narongchai (2008) estudiaron en el norte de Tailandia 499 cuerpos autopsiados y concluyeron que el peso de los órganos que han sido considerados en el presente estudio se incrementaba en los hombres en relación a una edad mayor, al peso y a la altura del cuerpo. También, la influencia del trabajo muscular sobre el desarrollo de corazón es considerable y esto se comprueba en el trabajo manual y en el atleta, que tienen un corazón más voluminoso que el del individuo sedentario (Testut \& Latarjet).

En nuestro estudio, el peso del corazón en relación al IMC se incrementó en alrededor de un $15 \%$ en ambos grupos étnicos y en ambos sexos, considerando un índice de masa corporal de 18-25 hasta 30-35.

En estudio llevado a cabo en la India, Prakash et al. (2013) indicaron que el peso promedio del corazón en hombres fue de 270, $3 \mathrm{~g}$ y en mujeres de 204,4 g y estos valores fueron positivamente correlacionados al peso del cuerpo, siendo constante o incrementado con el aumento de la edad. Estos promedios son inferiores a los obtenidos en nuestro estudio, pero coincidentes en la asociación de su aumento con la edad y peso corporal.

Como se puede apreciar, efectivamente hay un aumento en el peso del corazón respecto al aumento de la edad, señalando que, según Williams et al., el peso adulto de este órgano es alcanzado entre los 17 y 20 años. El índice de masa corporal también influye en el peso de este órgano. Además, se corrobora que el corazón tiene mayor peso en el hombre, fundamentalmente por causa de la diferencia de altura y peso del cuerpo. Se dice que el peso del corazón corresponde a un $0,45 \%$ del peso corporal en el hombre y de $0,40 \%$ en la mujer (Williams et al.).

Con respecto a los pulmones, Testut \& Latarjet indicaron que en el adulto, el peso absoluto varía de 900 a 1.300 g, como término medio son 1.100 g, 600 para el pulmón derecho y 500 para el izquierdo, valores semejantes a los descritos por Williams et al., $625 \mathrm{~g}$ para el pulmón derecho y 565 para el izquierdo. Sin embargo Gardner et al., han mencionado valores menores, 375-550 g para el pulmón derecho y 325-450 g para el pulmón izquierdo, esto considerando hombres adultos de 20 a 30 años de edad. Molina \& DiMaio (2012b) registraron un peso promedio para el pulmón derecho de $445 \mathrm{~g}$ y para el izquierdo de $395 \mathrm{~g}$.

Los registros generales de nuestra serie, en ambos grupos étnicos, han arrojado pesos promedios menores en el pulmón derecho que los dos primeros autores mencionados y similares en los registros del pulmón izquierdo, pero superiores a los de Gardner et al. y Molina \& DiMaio (2012b), para ambos pulmones. 
Considerando la variable sexo, Prakash et al. registraron un peso promedio para el pulmón derecho de 446,6 g y $334 \mathrm{~g}$, para hombres y mujeres, respectivamente, y, para el pulmón izquierdo de 477,9 $\mathrm{g}$ en hombres y de $344,4 \mathrm{~g}$ en mujeres. Encontraron que el peso del pulmón esta correlacionado al peso del cuerpo en las mujeres, no así en el hombre. El estudio de Molina \& DiMaio (2012b) mostró que en hombres hubo una asociación insuficiente entre el peso de estos órganos y el IMC, corroborando lo señalado por los autores previamente mencionados. Sin embargo, Mathuramon et al. (2009) han indicado que existe relación entre el peso de estos órganos y el peso del cuerpo.

En el estudio realizado en Corea del Sur por Kim et al. se obtuvo un peso promedio de $645,6 \mathrm{~g}$ para el pulmón derecho en el hombre y de 485,4 g para la mujer, mientras que en el el pulmón izquierdo fue de 559,1 g y 417,6 g, respectivamente.

Los resultados obtenidos en nuestro estudio muestran que respecto a la edad, en los hombres del grupo étnico Mapuche el peso de estos órganos disminuye levemente entre la edad de 20-29 años y los 50-59 años, en cambio en el grupo no-Mapuche su peso se incrementa en alrededor de $8 \%$. En las mujeres del grupo no-Mapuche, esta variable disminuye en el mismo porcentaje en ambos pulmones. No ocurre lo mismo en el caso de las mujeres en el grupo étnico Mapuche, donde los pulmones aumentaron de peso por sobre un $40 \%$. Este aumento ocurre tempranamente, desde el grupo de 20-29 años hasta la faja etaria de 30-39 años, donde se observa que ambos pulmones incrementan su peso promedio en alrededor de $35 \%$.

Relacionando el peso de estos órganos con el IMC, no se produce un incremento importante entre individuos de IMC normal con los individuos que presentan sobrepeso o un grado de obesidad 1, inclusive en el grupo Mapuche los valores se mantienen constantes, al igual que en los hombres del grupo no-Mapuche, en cambio en las mujeres de este grupo, los valores disminuyen desde un IMC normal al de Obesidad tipo 1 en aproximadamente $15 \%$.

Los resultados obtenidos son un aporte significativo al conocimiento del peso del corazón y pulmones en individuos chilenos, considerando dos grupos étnicos del Sur de nuestro país y la relación de estos órganos con la edad, sexo e IMC.

OLAVE E.; TORREZ, J. C.; RIQUELME, N.; IBACACHE, L. \& BINVIGNAT, O. Weight of the heart and lungs, with regard to sex, ethnic group, age and body mass index in Chilean individuals. Int. J. Morphol., 32(3):866-870, 2014.

SUMMARY: Several factors can influence the weight of the thoracic and/or abdominal viscera. In order to know the average weight of the heart and lungs of Chilean individuals, information of these organs was obtained from 775 necropsies of fresh cadavers, between 2009-2013, considering ethnicity (non-Mapuche and Mapuche), age, sex and body mass index. We studied organs without apparent injury or disease. When considering age, the average heart weight showed an increase in both ethnic groups and both sexes. This increase was similar in male individuals from both ethnic groups, with a value of $13 \%$. In female individuals there was an increase of $36 \%$ in the Mapuche ethnic group, while that of nonMapuche was $8 \%$. With regard to the lungs, there was no increase in their average weight in male individuals of Mapuche group, in females however, the increase was about 40\%. Among the group of 20-29 years and 30-39 y, both lungs increased their average weights at $35 \%$. In the non-Mapuche group, in both sexes, the average weight of these organs increased by less than $10 \%$. These results are compared with other population groups in the world and are a contribution to morphological knowledge of the thoracic viscera.

KEY WORDS: Anatomy; Thorax; Visceral weight; Heart; Lungs.

\section{REFERENCIAS BIBLIOGRÁFICAS}

de Suárez, C. \& Avilán, R. J. M. Pesos en corazones normales en Venezuela. Gac. Méd. Caracas, 112(1):42-8, 2004.

Gardner, E.; Gray, D. \& O’Rahilly, R. Anatomía. Estudio por regiones del cuerpo humano. $2^{\mathrm{a}}$ ed. Barcelona, Salvat, 1977.
Hodkinson, I.; Pomerance, A. \& Hodkinson, H. M. Heart size in the elderly: a clinicopathological study. J. R. Soc. Med., $72(1): 13-6,1979$.

Kim, Y-S.; Kim, D-I.; Cho, S. Y.; Kim, M. H.; Yang, K. M.; Lee, H. Y. \& Han, S-H. Statistical analysis for organ weights in Korean adult autopsies. Korean J. Anat., 42(4):219-24, 2009. 
OLAVE, E.; TORREZ, J. C.; RIQUELME, N.; IBACACHE, L. \& BINVIGNAT, O. Peso del corazón y pulmones en relación al sexo, grupo étnico, edad e índice de masa corporal en individuos chilenos. Int. J. Morphol., 32(3):866-870, 2014.

Kitzman, D. W.; Scholz, D. G.; Hagen, P. T.; Ilstrup, D. M. \& Edwards, W. D. Age-related changes in normal human hearts during the first 10 decades of life. Part II (Maturity): A quantitative anatomic study of 765 specimens from subjects 20 to 99 years old. Mayo Clin. Proc., 63(2):137-46, 1988.

Mathuramon, P.; Chirachariyavej, T.; Peonim, A. V. \& Rochanawutanon, M. Correlation of internal organ weight with body weight and length in normal Thai adults. J. Med. Assoc. Thai., 92(2):250-8, 2009

Molina, D. K. \& DiMaio, V. J. Normal organ weights in men: part I-the heart. Am. J. Forensic Med. Pathol., 33(4):362-67, 2012a.

Molina, D. K. \& DiMaio, V. J. Normal organ weights in men: part II-the brain, lungs, liver, spleen, and kidneys. Am. J. Forensic Med. Pathol., 33(4):368-72, $2012 b$.

Narongchai, P. \& Narongchai, S. Study of the normal internal organ weights in Thai population. J. Med. Assoc. Thai., 91(5):74753,2008 .

Prakash, C.; Deopa, D. \& Thakkar, H. K. Study of internal organ weight and its correlation to body weight in kumaon region of uttarakhand. J. Indian Acad. Forensic Med., 35(1):29-32, 2013.

Ruiz-Montero, P. J.; Castillo-Rodríguez, A.; Mikalacki, M.; Nebojsa, C. \& Korovljev, D. Previous anthropometric measures in adult and elderly Serbian women to physical and educational program of pilates and aerobic. Int. J. Morphol., 31(4):12638, 2013.

Sheikhazadi, A.; Sadr, S. S.; Ghadyani, M. H.; Taheri, S. K.; Manouchehri, A. A.; Nazparvar, B.; Mehrpour, O. \& Ghorbani, M. Study of the normal internal organ weights in Tehran's population. J. Forensic Leg. Med., 17(2):78-83, 2010.

Smith, H. L. Relation of weight of heart to weight of body and of the weight of heart to age. Am. Heart J., 4:79-93, 1928.

Testut, L. \& Latarjet, A. Tratado de Anatomía Humana. Barcelona, Salvat, 1969. V. 3.

Williams, R. L.; Warwick, R.; Dyson, M. \& Bannister, L. H. Gray Anatomía. 37a ed. Rio de Janeiro, Guanabara-Koogan, 1995.

\author{
Dirección para Correspondencia: \\ Dr. Enrique Olave \\ Facultad de Medicina \\ Universidad de La Frontera \\ Casilla 54-D, Temuco \\ CHILE
}

Email: enrique.olave@ufrontera.cl

Recibido : 10-05-2014

Aceptado: 22-06-2014 